\title{
Novel Long-Acting Ropeginterferon Alfa-2b: Pharmacokinetics, Pharmacodynamics, and Safety in a Phase I Clinical Trial
}

\author{
Yi-Wen Huang ${ }^{1}$, Chung-Wei Tsai ${ }^{2}$, Albert Qin ${ }^{2}$, Jane Fang ${ }^{3}$, Chingleou Teng ${ }^{2}$, and Richard \\ Larouche $^{4}$ \\ ${ }^{1}$ National Taiwan University College of Medicine \\ ${ }^{2}$ Pharmaessentia Corp. \\ ${ }^{3}$ Athenex Inc \\ ${ }^{4}$ Syneos Health Inc
}

August 13, 2021

\begin{abstract}
AIM Ropeginterferon alfa-2b is a new site-specific conjugated $40 \mathrm{kDa}$ branched polyethylene-glycol recombinant interferon (IFN). The aim of the study was to determine its safety, pharmacokinetics (PK) and pharmacodynamic (PD). METHODS Ropeginterferon alfa- $2 \mathrm{~b}$ was evaluated first in human in 48 healthy male volunteers after a single dose subcutaneous injection by either $24,48,90,180,225,270 \mathrm{mcg}$ of the product or $180 \mathrm{mcg}$ of marketed pegylated (peg)-IFN alfa-2a. Within each dosing group, 6 subjects received ropeginterferon alfa- $2 \mathrm{~b}$ and 2 subjects received peg-IFN alfa-2a. RESULTS Dose-related increases in ropeginterferon alfa-2b PK parameters (Cmax, AUC, and AUC0-t) were observed over the dose range 24 to $270 \mathrm{mcg}$. The geometric mean values for these PK parameters of ropeginterferon alfa- $2 b$ were higher than that of peg-IFN alfa- $2 a$ at the $180 \mathrm{mcg}$ dose level of $176 \%, 166 \%$, and 182\%, respectively. Mean PD parameters (Emax, Tmax, and AUC0-t) for ropeginterferon alfa-2b increased with dose for both biomarkers neopterin and 2', 5'-OAS. Ropeginterferon alfa-2b has similar PD profiles as peg-IFN alfa-2a. The treatment related adverse events are similar between the two study drugs, but the overall incidence was numerically lower for ropeginterferon alfa-2b (83\%) than peg-IFN alfa-2a (100\%) at the 180mcg dose level. CONCLUSIONS Single subcutaneous dose of Ropeginterferon alfa- $2 \mathrm{~b}$ of up to $270 \mathrm{mcg}$ is safe and well tolerated. It displays dose related increase in PK and PD parameters, potentially less frequent injection, and better safety profiles. Ropeginterferon alfa- $2 \mathrm{~b}$ is being developed for diseases in which previous peg-IFN use has been limited by side effects.
\end{abstract}

\section{Novel Long-Acting Ropeginterferon Alfa-2b:}

Pharmacokinetics, Pharmacodynamics, and Safety in

a Phase I Clinical Trial

Yi-Wen Huang, ${ }^{1,2}$, 3, 4,* Chung-Wei Tsai, ${ }^{4}$ Albert Qin, ${ }^{4}$ Jane Fang, ${ }^{5}$ Ching-Leou Teng, ${ }^{4}$ Richard Larouche ${ }^{6}$

\section{Affiliations:}

${ }^{1}$ Division of Gastroenterology and Hepatology, Department of Internal Medicine, School of Medicine, College of Medicine, Taipei Medical University, Taipei, Taiwan

${ }^{2}$ Division of Gastroenterology and Hepatology, Department of Internal Medicine, Taipei Medical University Hospital, Taipei, Taiwan

${ }^{3}$ School of Medicine, National Taiwan University College of Medicine, Taipei, Taiwan

${ }^{4}$ PharmaEssentia Corporation, Taipei, Taiwan 
${ }^{5}$ Athenex Inc., New York, USA

${ }^{6}$ Syneos Health, Montreal, Québec, Canada

*Correspondence to:

Yi-Wen Huang, M.D., Ph.D.

Associate Professor,

Division of Gastroenterology and Hepatology,

Department of Internal Medicine,

Taipei Medical University Hospital \&

School of Medicine, College of Medicine,

Taipei Medical University

No. 252, Wuxing St, Xinyi District,

Taipei City, 110, Taiwan

Tel.: +8862-27372181

E-mail:yiwenhuang@tmu.edu.tw

\section{Running Head}

Ropeginterferon alfa-2b Phase I Study in Healthy Subjects

Keywords: interferon; pegylated interferon; phase 1; first in human

\section{Word count}

Word count: 3,759, Table count: 4, Figure count: 2.

\section{Data availability statement}

The data that support the findings of this study are available on request to the corresponding author. The data are not publicly available due to privacy or ethical restrictions.

\section{Funding statement}

This study was funded by the PharmaEssentia Corp. The interpretation and conclusions in this study are based on the authors' view.

\section{Authorship statement}

CLT proposed the concept for study and contributed to the design of the study. JF participated in design of the study, preparation of the protocol, case report form, acted as the medical monitor, review of data/analyses, internal and external presentation and preparation of clinical study report. YWH, CWT and $\mathrm{AQ}$ contributed to the writing of the manuscript and interpretation of the data. RL was principal investigator to conduct study and contributed to acquisition, analysis and interpretation of data. All authors reviewed and approved the final manuscript.

\section{Conflict of interest disclosure}

Jane Fang served as a clinical research consultant for PharmaEssentia Corp. in this study. The other authors have no conflicts of interest to declare.

\section{Ethics approval statement}


The study was conducted in accordance with the provisions of the Declaration of Helsinki and its amendments, US Food and Drug Administration (FDA) guidance, and principles of Good Clinical Practice (GCP) from the International Conference on Harmonization (ICH) guidelines. The protocol and informed consent form were reviewed and approved by an Institutional Review Board (IRB) prior to the screening or enrollment of any study participant.

\section{Patient consent statement}

The purpose of the study was explained to the patients before they provided their written informed consent. Patients were provided with copies of their signed informed consent forms. At the study center, the clinical staff was available to patients before they entered the study and throughout their participation in the study to answer questions about the study. Patients were informed about any new development during the study that might have influenced their continued participation in the study.

\section{Permission to reproduce material from other sources}

Not applicable

\section{Acknowledgement}

The authors thank the patients, their families, and the study investigator who took part in this study. In addition to the authors, the following investigators participated in the study: Jean-Jacques Barbeau, Pierre Bélanger, Caroline Champagne, Benoit Deschamps, Lief Destrade Sosa, Martine Gobeil, Marie-Christine Godin, Geneviève Ostiguy, Idania Teresa Garcia Del Sol, François Saint-Maurice, Luc Tremblay, Susan Mcvety and Isabelle Boisclair. The author also thanks Jean Couture, Marie-Eve Coulombe and Amelie Racicot for the statistical analysis of the data.

\section{Clinical Trial Registration}

This study was submitted to US FDA for IND (IND number: 105653) but not registered in ClinicalTrial.gov.

\section{What is already known about this subject?}

The marketed pegylated interferons have limited clinical use due to their side effects, which might also limit the optimal dose for better efficacy.

Pegylated interferons have potential roles in unmet medical needs and in broad therapeutic areas.

\section{What this study adds?}

Ropeginterferon alfa- $2 \mathrm{~b}$ is a novel pegylated interferon with superior pharmacokinetic parameters than the marketed pegylated interferon product, allowing biweekly injection.

Ropeginterferon alfa-2b has better safety profiles than the marketed pegylated interferon product.

\section{Hosted file}

A09-102 Study_Phase I_20210503.docx available at https://authorea.com/users/430446/articles/ 533942-novel-long-acting-ropeginterferon-alfa-2b-pharmacokinetics-pharmacodynamics-andsafety-in-a-phase-i-clinical-trial 
Fig. 1.

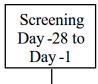

Screening
Day-28 to
Day-1

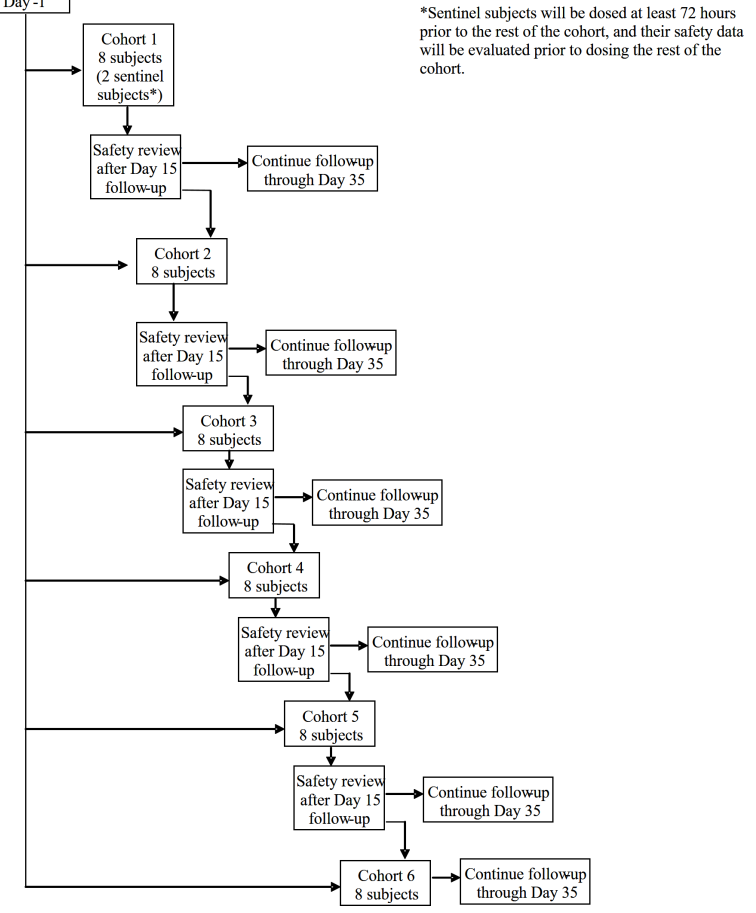

Fig. 2.

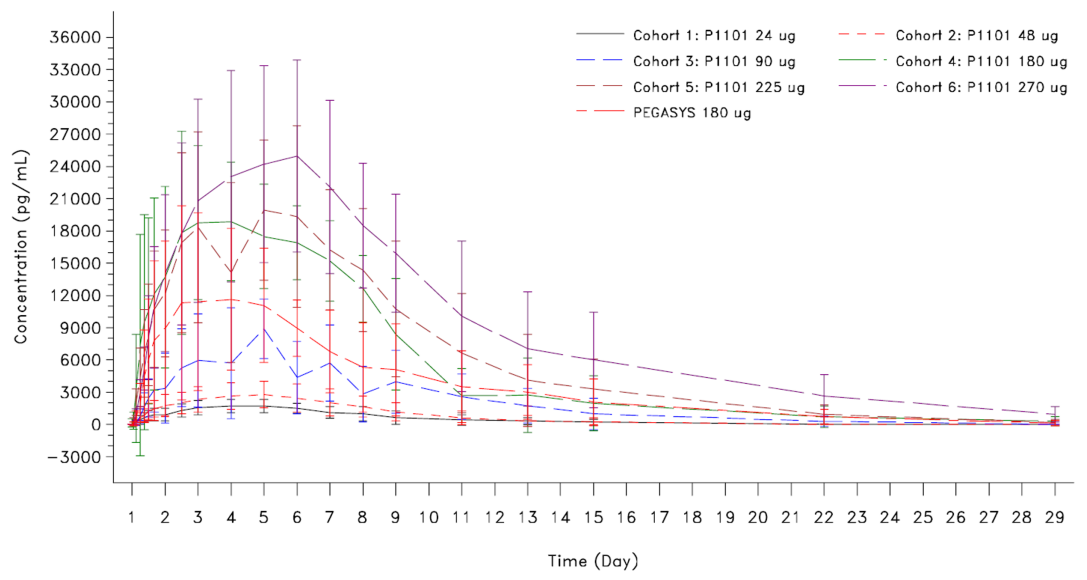

